International Journal of Research in Advent Technology, Vol.7, No.4, April 2019

E-ISSN: 2321-9637

Available online at www.ijrat.org

\title{
Comparative Study Of Traditional And Evolutionary Approaches For Cloud Service Selection
}

\author{
Preeti Sirohi ${ }^{1}$, Amit Agarwal ${ }^{2}$, Piyush Maheshwari ${ }^{2}$ \\ ${ }^{1}$ Ph.D Research Scholar, University of Petroleum and Energy Studies, Dehradun \\ ${ }^{2}$ Department of Computer Science, University of Petroleum and Energy Studies, Dehradun \\ ${ }^{2}$ Department of Computer Science, Amity University, Dubai
}

\begin{abstract}
The increasing demand of cloud attracts more and more cloud providers and cloud consumers. Due to the diversity of available cloud service in the market puts up a challenge for the user which service to take from the provider. The service selection and cloud ranking is considered challenging and therefore there should be some efficient approach which can select and rank cloud service according to the customized requirements of the user. Traditional approaches and evolutionary approaches are proposed by several researchers in this area for facilitating user in providing solution related to their service selection problem. The paper explores and performs a literature review of common and most used Multi criteria decision making methods and evolutionary approaches to evaluate the advantage or the weakness in the proposed approaches. The analysis of both traditional and evolutionary approaches performed in this paper provides a clear picture of how these methods can be used in the particular situations.
\end{abstract}

Index Terms: Cloud Computing; Ranking; Multi-Criteria

\section{INTRODUCTION}

Information and Communication Technology (ICT) has gained its popularity these days and where cloud technology has made computing as a service model where cloud services can be taken as utility services like electricity, water, etc. . Cloud Computing service model facilitates the user by providing access to the offered services, application and resources through internet. Cloud technology gives an environment to its users where they can access and use resources in lease from anywhere in the world [1].

According to NIST definition "Cloud computing is a model for enabling convenient, on demand network access to a shared pool of configurable computing resources (e.g. networks, servers, storage, applications and services ) that can be rapidly provisioned and released with minimal management effort or service provider interaction [2]. The cloud service selection is based on various attributes and their sub-attributes which are important for selecting services. The attributes are helpful in deciding which cloud services should be taken in order to meet the requirements. Few of the attributes are discussed here :-First Attribute is the security and privacy of various services and resources which is provided through various authentication and encryption techniques used in cloud [3] . Second attribute is the cost model which is the payment system for the cloud and the cost will depend on the type of the resources used by the consumers. Pricing model for cloud can be either pay per use [4] model or dynamic pricing model [5]. Third attribute is $\mathrm{On}$ demand self service. which allows the allocation and de-allocation of the resources according to the requirement [3]. Fourth attribute is Multi-tenancy in which there are multiple consumers who can use the same resources [6]. Sixth attribute Ubiquitous network access which confirms that all the devices which are connected in the network can easily access the cloud services [7].

Cloud computing is based on two main technologies which also helps in achieving the above characteristics. The first technology is Service oriented Architecture (SOA) [2] in which the complete task is divided into equal sub-task performing independent functions. These sub-tasks are known as "service" offered to the customers [8].. Another technology for cloud technology is "Virtualization". The various computing resources are represented to virtual devices which is used by user for carrying out computing tasks and these resources can be accessed through internet. A Cloud computing environment is based on three major element which are clients, distributed servers and datacenter. In cloud each component has an element with some specific functions [9]. Clients are the medium through which user interacts with the cloud environment which can be distinguished into thin client, thick client and mobile client [10]. Datacenter is a assembly of servers organizing different applications. An end user linked to the datacenter to 
International Journal of Research in Advent Technology, Vol.7, No.4, April 2019

\section{E-ISSN: 2321-9637}

Available online at www.ijrat.org

take different applications. A datacenter may situated at a great distance to the clients. Now-a-days a method called virtualization is used to install software that permits multiple instances of virtual server applications [11]. Distributed servers are the parts of a cloud which are available all through the Internet, hosting various applications. But during using the application from the cloud, the user will think that he is using this application [12].

\section{EXISTING CLOUD SERVICE SELE TION TECHNIQUES}

In this section, the techniques of cloud service selection and ranking are studied in detail under various categories mentioned in the basic process of cloud service selection and ranking. The categorization of service selection under various techniques will make an outstanding contribution of the existing research.

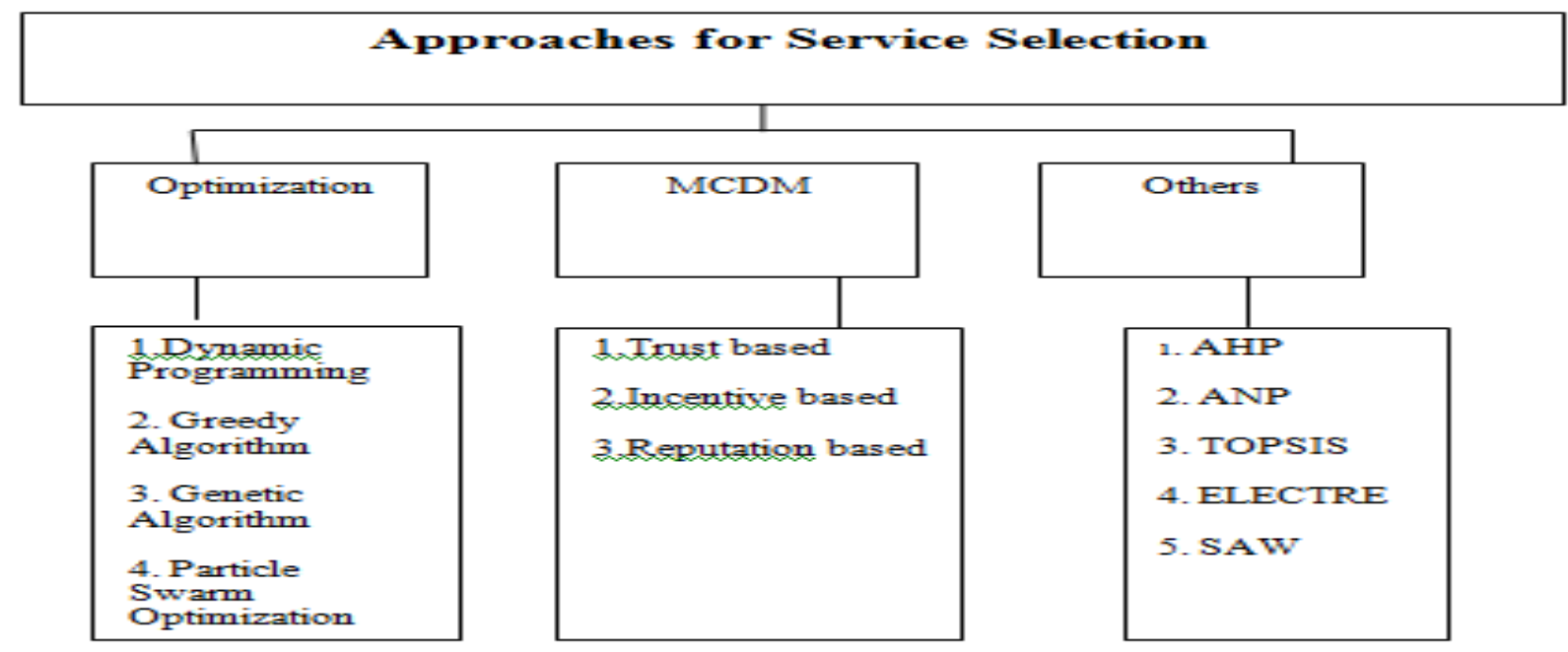

Fig 1: Taxonomy of various approaches of service selection

\subsection{MCDM-Based Cloud Service Selection}

Decision making in cloud service selection is identifying the services that best fits the customer requirements. Multiple -Criteria decision making (MCDM) [13] is the common technique and is used when the number of the decision criteria and the number of alternatives is finite. The popular MCDM techniques include the Analytic Hierarchy Process/ Analytic Network Process (AHP/ANP) [14] , Weighted Sum [15], Technique for Order of Preference by Similarity to Ideal Solutions (TOPSIS) [16], ELECTRE [17] , Optimization based service selection includes dynamic programming [18], greedy algorithm [19], Particle Swarm Optimization [20] and genetic algorithm [21]. Various other approaches such as Trust based [22,23] and incentive based approaches[24].

\subsubsection{Analytic Hierarchy Process (AHP)}

AHP is a decision making technique which help the decisions maker to find the best suited alternative from the list of alternatives keeping in mind the requirement of the decision maker. It is also known as structured technique which helps in analyzing the best from the complex decisions. An AHP ranking process is divided into three parts decomposition, comparative judgment and synthesis $[\mathbf{2 5 , 2 6 ]}$. The hierarchical designing of the framework in AHP helps the decision maker to systematically evaluate the various alternatives and comparing them for finding the best alternative. Fig 4 shows the AHP hierarchy which aims to achieve goal on the basis of the criteria 1 , criteria 2 ,

criteria 3 and criteria 4.. Second part is comparative judgment part pair wise comparison of the alternatives is done. The third part shows the priorities of the alternatives which are synthesized to find the best alternative.

The analytic network process (ANP) [26] is also used in multi criteria decision making, the criteria and the alternatives are structured into network form. ANP technique is used in decision making where the problem can not be structured hierarchical. Figure 5 shows the ANP network, here the criteria and the alternative are shown in the form of clusters rather than hierarchies. To show the relationship between the criteria and the alternatives arrow indicators are used. The elements in one cluster are shown as 
International Journal of Research in Advent Technology, Vol.7, No.4, April 2019

\section{E-ISSN: 2321-9637}

Available online at www.ijrat.org

interdependence, the dependence between two cluster are shown as outer dependence.

Godse and Mulik in [27], proposed an AHP based technique for cloud service selection. The selection is based on SaaS cloud. The author proposed SaaS product selection model consist of five attributes including Functionality, Architecture, Usability, Vendor Reputation and Cost. There are also sixteen sub attributes including contact and activity management, opportunity management, sales performance management, Sales analysis, Integration, Scalability, Reliability, Security, User Interface, Help, Support for mobile device, Offline support, Number of clients , Brand Value, Annual Subscription, One time implementation cost. The weights of each attribute and sub attribute are calculated, pair wise one-one comparison is done which gives the scoring of each attribute and thereby ranking of each attribute is calculated.

In [28] Raed et al discovered the selection and aggregation of QoS parameters of cloud services, facilitating the ranking of IaaS and PaaS services according to the user requirements. The author proposed the mechanism which will map QoS requirement of cloud services to the SaaS provider which will match the requirement which in turn matches to the best IaaS service offering optimal QoS guarantees. AHP method is used to model hierarchical model the QoS specifications.

Gonclaves et al. in [29] talked about the importance of the architectural design selection of cloud as it will increase the cost and also will not be able to fulfill the minimum required performance. The author proposed an AHP based cloud selection which relies on non functional parameters including efficiency, cost and scalability based on multi criteria optimization method. The proposed approach of the author is implemented on word press which is deployed in Amazon cloud. In [30] Menzal et al. proposed the Multi-Criteria Comparison (MC2)2 framework for cloud IaaS selection. The framework helps the user to identify which infrastructure would be best suited for their requirements.

The (MC2)2 distinguish the infrastructure alternatives in terms of cost, benefits, opportunities and risk. The framework (MC2)2 can be taken in various decision making scenarios in information technology infrastructures.

In [31] , Sun et. al presented a new framework based on fuzzy decision approach, the proposed framework will improve the service selection techniques existing in cloud. Fuzzy Ontology based model is developed to reduce the uncertain relations among various objects defined in database and used for service selection. An AHP based multi criteria technique is used to rank the services in cloud.
Evaluation of the proposed method is done to evaluate its performance. Boutkhoum in [32] proposed a methodology which is based on the Fuzzy Analytic Hierarchy Process(FAHP) and PROMETHEE (Preference Ranking Organization method for Enrichment Evaluations) for helping in analyzing and ranking the best cloud which removes the issues related to the big data and cloud technology. The Fuzzy AHP technique helps in assigning weights for evaluation of the criteria while PROMETHEE method will evaluate the weight assigned and will rank the decisions alternatives.

Khowfa and Silasai in [33] proposed a novel approach using Association Rules technique and AHP method. Association Rules is commonly used in data mining for finding out relations among various items in the database. AHP is the multi criteria decision making method for comparing and ranking of cloud services, The combination of association technique and AHP will evaluate and select appropriate services.

\subsubsection{Technique For Order Of Preference By Similarity To Ideal Solution (TOPSIS)}

TOPSIS technique was developed by Hwang and Yoon in 1981. The technique considers the ideal solution and find the best alternative by selecting the alternative that is near to the ideal solution and at the same time it is farthest from the anti-ideal solution [34] . Vector Normalization is used for normalization of decision matrix and then ideal and anti-ideal solutions are identified keeping in view the normalized decision matrix [35]. Cloud Service Selection Using Multi-Criteria Decision Analysis. The TOPSIS method does not consider the relative importance of the attributes but only take into consideration the ideal solution and the non ideal solution. The TOPSIS technique along with fuzzy applied for calculating weights of different criteria and later service selection is done based on the above technique.

In [36] Lo et. al. discussed about fuzzy TOPSIS method in which the predetermined linguistic variables which is parameterized by triangular fuzzy numbers which helps in calculating weights of different criteria and the rating of alternate service are calculated.. Rai and Kumar in [37] uses the traditional service selection method based on the criteria weights according to user demand. Different data centres are used for providing different computing environments also referred as the cloud service providers and are responsible for publishing services which are

stored in database. The other part is the users of the services who give the requirement of the services according to their priority. The broker is responsible 
International Journal of Research in Advent Technology, Vol.7, No.4, April 2019

\section{E-ISSN: 2321-9637}

Available online at www.ijrat.org

identifying the suitable services according to users requirements.

\subsubsection{Elimination and Choice Expressing Reality (ELECTRE)}

This method is one of the outranking methods [38] of MCDM approach. Several versions of ELECTRE methods were released like ELECTRE I -VI, ELECTRE IS, ELECTRE TRI. There are two set of parameters in ELECTRE one is the importance coefficient and other is veto fresh ELECTRE performs a pair wise comparison between the set of alternatives and eliminate the alternatives which are less fitting into the criteria thereby leaving a small set of alternatives. The ELECTRE technique can be useful for both subjective and objective parameters. In order to construct the partial raking of the set of alternatives left after elimination it concordance and discordance indices are applied [39] .

In [40], Abdolreza Yazdani et al. used the ELECTRE technique for identifying the risk components in the tunneling project. The research is done in two phases, in the phase one all the potential risky parameters are defined and in phase two an order is established among them. As the tunneling system is complicated and involved lot of uncertainties therefore Fuzzy ELECTRE is used. Parameters such as damages, accidents of machinery and unsafe working conditions are prioritize in high risk and delay in project completion and failure of equipments are ranked lower.

Silas et al in [41] proposed a middleware for cloud service selection known as SSM_EC. According to the user preference related to QoS parameters and service description information collected from the provider, the concordance index and discordance index is evaluated also the credibility degree is calculated. Concordance index will give the truthfulness result according to the criteria set to calculate the relation between the candidates; the discordance will give the QoS difference between alternatives. Finally the credibility is calculated aggregating concordance and discordance index.

\subsubsection{Simple Additive Weighting Method (SAW)}

Simple additive weighting method [42] is also known as weighted linear combination method as it is based on weighted average is another multi criteria decision making technique. The alternatives are evaluated by the equation

$\mathrm{Ai}=\sum$ wjxij

where $\mathrm{Ai}$ is the ith alternative,

wj is the weight of the jth criterion, $x i j$ is the score of ith alternative with respect to the jth criterion [38].
Chou et. al. in [43], presented a noval fuzzy multiple attributes decision-making (FMADM) approach by using fuzzy approach with SAW method (FSAWS). Both the qualitative and quantitative attributes are taken to solve the problem related to the facility location under group decision criteria. The procedure used in FSAWS taken into account the decisionmaker idea with the final scores for each alternative locations which is derived by different group of decision-makers.

In [44], Saripalli and Pingali used Simple Additive Weighting (SAW) method to rank the alternatives by the values assigned to them and to find the best alternative. The author presented a MADMAC framework for the adoption of cloud services. The framework has three functions which will act as the decision areas known as cloud switch, cloud type and vendor choice. A modified Wide-band Delphi method is also proposed for accessing weights of each attribute with the help of an expert interview, the weights further helps in calculating relative rank. Upadhyay in [45] discussed about the problems faced while evaluating the cloud service performance. The paper talked briefly about cloud computing along with the importance of quality of service in cloud computing. The framework is proposed evaluation approach based on QoS parameters.

\subsection{Optimization Based Cloud Service Selection}

In Cloud service selection, optimization can be defined as finding the most suitable alternative or service for the user keeping in mind the reduced cost and high performance under the set criteria by maximizing the required parameters and minimizing the undesired parameters. Various Optimization techniques which are used in cloud service selection are classified into Integer Programming, dynamic programming s Genetic Algorithm, Hill Climbing algorithm, Ant Colony Optimization, Particle Swarm Optimization, Greedy Algorithm, etc. The literature survey from shows the above algorithm used in the study of cloud service selection.

\subsubsection{Dynamic Programming}

In [46], Tao $\mathrm{Yu}$ and Kwei-Jay Lin explored end to end QoS challenges in case of composite services and also the issues when the services are taken by QoS broker. The author also proposed the algorithm which will improve user defined utility functions while overcoming delay constraints. The author also proposed two different approaches the combinatorial approach for improving multiple choice knapsack problem and graph approach for finding shortest 


\section{International Journal of Research in Advent Technology, Vol.7, No.4, April 2019 E-ISSN: 2321-9637 \\ Available online at www.ijrat.org}

\section{International Journal of Research in Advent Technology, Vol.5, No.1, January 2017}

path. Khurana and Bawa in [47] talks about the problem the cloud consumer faces while selecting the cloud provider who can fulfill the requirements of the users. In reference to the above problem the author proposes the cloud service broker approach for selecting quality based the provider for cloud services.

Andrzej Gosconski and Michael Brock in [48] talked about cloud providers who try to offer services which have improved performance, resource consumption and scalability while ignoring other important services also the cloud providers do not have any common platform where they can publish the services and which can help the cloud consumers to automatically select the services. The author proposed a Resource Via Web Service (RVWS) framework which facilitates the publication of services and ease the user for discovery and selection of the services. The framework will reduce the time and effort of cloud users to locate and evaluate the services but can go on the platform and can select the services required.

Maruthi and Shanthi in [49] proposed dynamic cloud service selection (DCS) in the multi cloud environment. DCS uses the cloud service broker method by keeping in mind the performance and cost of the services. The proposed DCS approach used the adaptive learning method for performing the service selection in cloud and at reduced cost. The process takes three layers into consideration namely user layer, cloud broker layer and cloud service layer for cloud service selection process.

\subsubsection{Greedy Algorithm}

In [50], Zibin Zheng et. al. discussed that the performance of cloud services are helpful to the users in selecting the right services. The author proposed a cloud ranking framework which will provide QoS values and will also reduce cost and time in finding the best services. Greedy algorithms are used to build two ranking prediction methods namely CloudRank 1 and CloudRank 2. These two ranking methods will predict QoS ranking. To do the study extensive real time experiments were conducted in order to check the accuracy of the cloud ranking algorithm and also compared it with other existing ranking algorithm. The proposed algorithm was found more efficient as compared to the other ranking algorithm.

In [51] , Qiang He et. al. proposed novel techniques for supporting service selection in multi-tenant SaaS. The technique will help the SaaS developers to understand QoS requirements for SaaS which will also help in achieving various optimization goals at the same time meeting cloud users QoS
requirements.In order to meet the above criteria MSSOptimiser (multi tenant SaaS optimizer) is introduced which will help in effective service selection in SaaS. In large scenarios when the number of user increases and all have different QoS requirement then the system

will be complex, in order to overcome the issues in case of large scenarios greedy algorithm will be introduced in order to help the developers to find an optimal solution. After experimental study it was finalized that MSSOptimizer is more efficient as compared to other approaches.

\subsubsection{Particle Swarm Optimization}

In [52], Wang et. al. talked about the importance of Quality of Service in web service selection and composition for any business applications. The author proposed a fast Cloud based web services (CWS) composition approach which adopts skyline operator to reduce unneeded CWS parameters. The Particle Swarm Optimization method is then used to select parameters which will be aggregated to form composite service. For experimental evaluation real dataset is used and the result showed that the proposed approach was better and efficient for CWS composition.

Kang in [53] proposed a strategy known as PSOGODSS (global optimization of dynamic Web service selection based on PSO) algorithm for improving QoS in web service selection. PSOGODSS will help in dynamic web service selection and web service composition into multi-objective service composition keeping in mind global QoS optimization. The multi-objective service composition will later changed into a single-objective problem. Experimental evaluation of the algorithm results in shows that PSO-GODSS is an efficient and feasible algorithm and is better than other algorithms which are used in similar web service selection and composition.

In [54], Yang et. al. studied various cloud service selection and composition approaches and identified that the efficiency in service selection is still a challenge. A cloud service selection model is introduced in the research which will help in evaluation of the services from three different perspective which are functional, non functional and transactional dimensions. The model is based on the concurrent service request from the user and service association problem. The novel coding strategy and a hybrid particle swarm optimization algorithm is designed which is composed of crossover and mutation operators of the genetic algorithm. The experimental study done on the proposed algorithm showed that the proposed approach is very effective and feasible. 
International Journal of Research in Advent Technology, Vol.7, No.4, April 2019

\section{E-ISSN: 2321-9637}

Available online at www.ijrat.org

\subsubsection{Genetic Algorithm}

Ye et. al. in [55], discussed that in cloud environment the services can be classified into two categories one is application services in which services are selected and utility services in which scheduling is done. The paper proposed a genetic algorithm based service composition approach. QoS model is made which calculates QoS values on four attributes which are response time, price, availability and reputation. In order to calculate QoS values, QoS constraints are also considered which are of two types local constraints and global constraints. The analysis of the genetic algorithm is done through comparison with other algorithms.

In [56], Klein et. al. proposed a novel network aware service composition approach in cloud computing. The approach is classified into three phases which are designing of network model in which network latency is calculated for the user which are in same location or different location. In order to calculate network latency already existing network model is taken and it is enhanced. Services which offer low network latency is preferred over the others. The second contribution is network aware QoS computation in which two QoS parameters such as latency and transfer rate is taken into account for computation of network QoS. The third contributions is designing if Network-aware Selection Algorithm which is based on genetic algorithm consisting of all the genetic operations such as initial generation, mutation and crossover. The experimental analysis done by comparing the proposed genetic algorithm with other algorithms and the result shows that latency of service composition which is computed by our algorithm is optimal and very less as compared to compared algorithm.

In [57], Maolin Tang and Lifeng Ai proposed Hybrid genetic algorithm for optimal web service selection and composition. The Hybrid genetic algorithm designed will overcome the dependency and conflicting constraints. The algorithm also solve the web service selection problem in the constrained environment and will improve the QoS solutions. The proposed algorithm is evaluated with two other algorithms and the result proved that the proposed hybrid genetic algorithm is better.

\subsection{Trust Based Cloud Service Selection}

Different cloud services are offered by different cloud providers, the dilemma of the cloud consumer is that if the cloud provider is providing all the cloud services which has been mentioned in SLA and the services offered is best and will meet the requirement of cloud consumer. Therefore how to select trustworthy cloud services and cloud service provider is still a challenge. Therefore various models are studied in the literature which shows that how the author has reduced the pressure of the decision maker in building trust and helps in selection of best services from a trustworthy cloud provider.

In [58], Pan et. al. proposed a cloud service selection model known as trust-enhanced similarity model which will help in improving trust between the provider and the consumer. This model will combine QoS of the cloud service, fills all the missing QoS and finally rank the cloud services eventually building trust in the cloud users. The cloud service selection model is divided into four parts. In first part author propose a trust modeling method in which a mutual trust degree between two users is build in general cases it is assumed to be equivalent. Secondly users experience are calculated and numerical values are assigned based on their experience on QoS values. Thirdly trust-enhanced similarity model is utilized to find all the similar trusted neighbors and finally ranking of the services is

done. After experimental analysis the proposed model is found more effective as compared to other approaches in cloud service selection and ranking of cloud services.

Josang et. al in [59] introduced the concept of trust from different perspective. The author talked about trust based selection in cloud computing as the trustworthiness between cloud providers and cloud users ie. both the parties can provide some rating to each other in order to derive trust and reputation. Various trust based approaches are discussed in the paper which have taken both the qualitative and quantitative attributes in drawing out the conclusion of trust and reputation between the cloud provider and cloud consumer. Qualitative parameters are evaluated by user feedback, expert's judgment and Quantitative parameters are evaluated through some performance evaluation approach of QoS.

$\mathrm{Wu}$ et. al. in [60], talked about reliability as an important parameter for ensuring trust. The novel unfair rating filtering method is proposed for revising already existing reputation system. The objective of the proposed method is filtering of all the ratings which are unfair and not important thereby increasing the overall performance through the market mechanism helping the service providers and the service consumers to go for the services of their choice. The proposed method shows an improved reputation revision system.

In [61], Ghosh et. al. proposed a trust framework known as SELCSP for computing the interaction risk between cloud provider and cloud consumer. The author discussed an importance of service level agreement (SLA) for ensuring guaranteed service quality and ensuring reliability of the cloud provider. 
International Journal of Research in Advent Technology, Vol.7, No.4, April 2019

$$
\text { E-ISSN: 2321-9637 }
$$

Available online at www.ijrat.org

The SELCSP framework both trustworthiness and reliability by estimating the risk of interaction between the provider and the consumer which is generally based on the collection of the trustworthiness computation through the consumer feedback taken or interview. $\mathrm{Hu} \mathrm{Ma}$ et. al. in [62] proposed a new cloud service interval neutroscopic set (CINS) based on time aware approach to evaluate the trustworthiness in the cloud service selection. The time aware problem is treated as multi criteria decision problem. The proposed CINS approach is used for ranking cloud services. The experimental evaluation of CINS is done using a real data sets and the effectiveness of the proposed approach is evaluated.

Supriya et. al in [63] talks about the importance of trust parameter between the cloud consumer and provider. Due to the increasing number of cloud services provider offering services, the challenge persist with the consumer on identifying the trustworthy provider. The author discussed various multi criteria decision making techniques which helps in ranking the cloud services and the providers. The combination of fuzzy technique along with analytic method is proposed for evaluating trust parameter in identifying from different cloud providers.

\subsection{Incentive Based Cloud Service Selection}

Incentive based service selection approach in cloud computing is like other service selection approach. The main aim of incentive based cloud service selection is to select the best services which fit according to the user requirements. Some of the incentive based literature is discussed below. Incentive based cloud service selection will increase the reputation of the cloud provider will improving trust for the cloud consumer. The cloud consumer can give a positive feedback on the services used by him which will improve the cloud providers reputation in the market.

Jurca and Faltings in [64], discussed in the previous work how the incentive based feedback system can be manipulated. The monetary benefits can be passed to the user providing the feedback on the online system and false report can be generated from there [65]. To stop the feedback from getting manipulated a collision- resistant feedback payment system is proposed. In the proposed system the several reports are generated from the feedback taken from the user and these reports can be used as a reference at the time of calculating the scores for the provider and when the proposed approach is compared with the existing approach the proposed approach is found better.
In [66], Papaioannou and Stamoulis proposed an approach for giving incentives for truthful feedback reporting in peer-peer system. The approach not only covers the user of the services but also transacting peers and the feedback is taken from both the parties. If there is any discrepancy in the feedback of any of the party then it shows that either or both the parties are lying. To overcome the above problem of the disagreement in the feedback a non- credibility metric is proposed which will give the punishment to whichever party is lying. In the proposed approach feedback is taken from each party after every single transaction in case if the report generated from the feedback gives similar result then the credibility values increases and the punishment goes down but in case if the feedback report are not similar then non-credibility valued are increased and so are the chances of punishment.

\subsection{Other MCDM Approaches For Cloud Service Selection}

Achar and Thilagam in [67] carried out the service selection in cloud with the help of three steps. The first step is the identification of the criteria according to Service Measurement Index (SMI) [68], the second step is the

determination of the weights of the criteria and the last step involves TOPSIS technique for ranking alternative services. The result of the study is evaluated through CloudSim toolkit [69].

In [70], Srivastava and Sorenson discussed QoS and also mentioned all the related literature about on QoS. The researchers who have worked on QoS has assumed that the user there is a linear relationship between the estimated QoS performance and the actual QoS performance but in real life situations it is not always correct. The author proposed a technique which will show the comparison between the user ratings and real QoS performance. The method used in the technique is mid-level splitting method [71]. The author in the research only took subjective parameters for the study. To assign weights to the attributes on the basis of customer preference Hypothetical Equivalents and In equivalents methods (HEIM) are used [72].

Sundareswaran et al. in [73] proposed a novel an architecture which is based on brokerage system. The author developed a exclusive technique for indexing. The proposed method will also help in supervising and managing the available information of cloud service provider. The $\mathrm{B}+$ tree method is used for indexing the cloud service provider and which will also helps in encoding the information about all the services and user requirements. The indexing architecture helps in storing the available cloud service properties and will also help the cloud user in 
matching and facilitating fast information retrieval. A greedy algorithm is also designed based on the CSP index which will provide ranking to various service provider and will improve cloud service selection. Various parameters and criteria are considered in order to improve the reliability and availability at the time of service selection in cloud.

Pandey and Daniel in [74] proposed a QoCS and cost based framework for service selection in cloud. The framework is based on trustworthiness and uses the fuzzy logic which helps the user in analyzing the cloud services on multi dimensional perspectives. Various parameters QoS parameters were used The costing of the services were the main criteria for the service selection. Experimental results also shows the better performance of the framework under various cost constraints.

\section{DISCUSSIONS}

The literature review is studied on the basis of four main approaches MCDM approach, Optimization approach and other approaches which takes combination of different approaches in order to get the result. There are various algorithms and framework studied under each approach . The main objective behind the research is to do a comprehensive survey of different work contributed by the researchers in the area of cloud service selection. The study is conducted keeping in mind the traditional approach and the evolutionary approach in the area of cloud computing. Various techniques and algorithm used in these approaches are studied on the basis of cloud deployment model, framework or algorithm proposed or presented and QoS parameters and implementation in the cloud environment. Different approaches and techniques are used for ranking the cloud services and cloud provider

which facilitates the decision maker in identifying the best services which can fit into the requirement of the user and business. Fig 2 shows a study related to different number of approaches studied in the literature. A detailed literature survey is done in which the research papers from 2003 onwards are covered and the limitations or the issues found in the existing survey is discussed in next section.

\subsection{Approaches Applied in Service Selection}

The applied and the proposed approaches used in service selection are divided into five distinct categories including MCDM approach, Optimization technique, Trust based service selection, Incentive based service selection and others. All the five different approaches are discussed in detail and are summarized in table 2 and figure 4 from which it can be inferred that MCDM approach is the used by the researchers while making decision in the service selection in cloud environment. Second highest approach used its applied approaches is Optimization Technique. Trust based selection and Incentive based selection approach are almost equal.

Table 2: Summary of total study done in reference to various service selection approaches

\begin{tabular}{|l|l|l|l|l|l|}
\hline Approach & MCDM & Optimization & Trust Based & $\begin{array}{l}\text { Incentive } \\
\text { Based }\end{array}$ & Others \\
\hline $\begin{array}{l}\text { No of Paper in } \\
\text { each Approach }\end{array}$ & 11 & 9 & 3 & 3 & 4 \\
\hline
\end{tabular}

\subsection{Quality of Service Parameters}

The quality parameters plays an important role in cloud service selection and study done in literature review done shows various QoS parameters which commonly appear are like trust, Throughput, Availability, Uptime, Risk, Efficiency, Reputation, Security and Scalability. The evaluation is done in table 3 bases on the number of repetitions of these parameters in the research papers. The more the number of repetitions shows that the parameter is considered more relevant. Some of the parameters like capability, correctness etc. are not studied in the literature. 
International Journal of Research in Advent Technology, Vol.7, No.4, April 2019

E-ISSN: 2321-9637

Available online at www.ijrat.org

Table 3: QoS Parameters in reference their importance in selection of services

\begin{tabular}{|l|l|}
\hline \multicolumn{1}{|c|}{ QoS Parameters } & No of Repititions in QoS \\
\hline Scalability & 4 \\
\hline Reliability & 3 \\
\hline Security & 3 \\
\hline Usabaility & 1 \\
\hline Reputation & 2 \\
\hline Cost & 8 \\
\hline Efficiency & 1 \\
\hline Response Time & 6 \\
\hline Risk & 3 \\
\hline Latency & 1 \\
\hline Uptime & 1 \\
\hline Capability & 1 \\
\hline Availability & 5 \\
\hline Accuracy & 1 \\
\hline Throughput & 1 \\
\hline Correctness & 1 \\
\hline Trust & 3 \\
\hline
\end{tabular}

\section{ISSUES IDENTIFIED IN CLOUD SERVICE SELECTION AND RANKING}

This section discusses few open challenges found in literature survey which is not studied sufficiently in the literature.

\subsection{Subjective Parameters Are Not Sufficiently Studied And There Are Not Enough Approaches For The Study.}

The service selection and ranking in cloud environment is based on QoS parameters which further classified into subjective and objective assessment. In order to efficiently select cloud services both the assessment parameters should be considered. The literature shows that objective parameters are more focused area of researchers in comparison to subjective parameters. In literature review of cloud service selection subjective and objective assessment should be classified separately and also an efficient technique should be designed which can handle the uncertainty and bias user feedback while carrying out assessment.

4.2 Consideration Of The Parameters Which Are Falling Into Subjective And Objective Assessment

Several criteria which are important and play a major role in service selection are studied separately. These criteria fall into both subjective and objective assessment. There are criteria which are also dependent on one another such

as cost and time [48]. Techniques which can assess such attributes need to be developed which can not only be assessed properly but also help the service selection based on mutually interdependent criteria.

\subsection{Multi-Tenancy Service Selection Evaluation Parameters Should Be Considered}

The literature review talks mostly about the single tenancy architecture in their service selection. The cloud technology is known for its multi-tenancy architecture and therefore it should be taken into account. The performance of the parameters in case of multi-tenancy will have an impact on the QoS of the overall service selected and therefore should be considered. The criteria like security, privacy, sharing of storage space, load balancing in case of multitenancy should be ranked through different techniques [69].

\subsection{Standards And Guidelines Should Be Defined To Evaluate Cloud Performance}

Due to increasing demand of cloud consumers for the customized services, there is no single standard which is followed by the cloud provider for offering the service. Therefore any common standard for identifying the cloud performance parameters attributes and quantitatively describing these attributes is believed impossible in practice. The 
International Journal of Research in Advent Technology, Vol.7, No.4, April 2019

$$
\text { E-ISSN: 2321-9637 }
$$

Available online at www.ijrat.org

literature does not show any commonly recognized taxonomy since all the cloud consumers have very different and customized views in cloud selection which is still an issue.

\subsection{Dynamic Cloud Service Selection Approach Is Not Studied Sufficiently.}

In cloud computing a cloud user want an assurance from the cloud provider that the service level agreement signed by both parties will be maintained over the long time. The performance of the services should be checked through some testing technique. The literature review mostly covers the QoS attributes through various approaches but there is not sufficient study have been seen on the decay process of the services.

\section{CONCLUSION AND FUTURE WORKS}

The comprehensive study and analysis after the literature review shows various approaches, frameworks and algorithms which are proposed by various researchers over a period of time. Table 1 and table 2 in the paper shows different approaches and their sub-approaches proposed by researchers and on which QoS parameters. In the literature multi-criteria decision making is most used topic of study, optimization problem has also area of interest for researchers. Incentive and trust based cloud service selection and ranking is analyzed in four different studies. The QoS parameters are also taken into account according to the researchers used in their study. It is found that cost

is and response time is widely used in the area of cloud selection and response time. The scalability and availability are also considered important and therefore involved in their study.

Different work proposed in the area of cloud is discussed in details and the role of QoS parameters helps in deciding the relevant services. The issues are also discussed and how they are impacting an overall process of cloud service selection. The literature review done in the research paper is through the proposed work and comparing various techniques and parameters used for service selection. The limitations in the existing literature discussed laid a foundation for future research directions. Authors can also explore multi- objective optimization approaches for service selection as the user have various objectives at time of service selection for which traditional approaches are not considered as an efficient method for cloud service selection.

\section{REFERENCES}

[1] Buyya, Rajkumar, et al. "Cloud computing and emerging IT platforms: Vision, hype, and reality for delivering computing as the 5th utility." Future Generation computer systems 25.6 (2009): 599-616.

[2] Mell, Peter, and Tim Grance. "The NIST definition of cloud computing." National Institute of Standards and Technology 53.6 (2009): 50.

[3] Lin, Wenmin, et al. "A QoS-aware service discovery method for elastic cloud computing in an unstructured peer-to-peer network." Concurrency and Computation: Practice and Experience 25.13 (2013): 1843-1860.

[4] Rackspace cloud.http://www.rackspacecloud.com/ Accessed on 20/June /2017.

[5] Mell, Peter, and Tim Grance. "The NIST definition of cloud computing." National Institute of Standards and Technology 53.6 (2009): 50.

[6] Zhang, Qi, Lu Cheng, and Raouf Boutaba. "Cloud computing: state-of-the-art and research challenges." Journal of internet services and applications 1.1 (2010): 7-18.

[7] Garg, Saurabh Kumar, Steve Versteeg, and Rajkumar Buyya. "Smicloud: A framework for comparing and ranking cloud services." Utility and Cloud Computing (UCC), 2011 Fourth IEEE International Conference on. IEEE, 2011.

[8] Murray, Paul. "Enterprise grade cloud computing." Proceedings of the Third Workshop on Dependable Distributed Data Management. ACM, 2009.

[9] Weinhardt, Christof, et al. "Cloud computing-a classification, business models, and research directions." Business \& Information Systems Engineering 1.5 (2009): 391-399.

[10] Mihailescu, Marian, and Yong Meng Teo. "Dynamic resource pricing on federated clouds." Proceedings of the 2010 10th IEEE/ACM International Conference on Cluster, Cloud and Grid Computing. IEEE Computer Society, 2010.

[11]Espadas, Javier, et al. "A tenant-based resource allocation model for scaling Software-as-a-Service applications over cloud computing infrastructures." Future Generation Computer Systems 29.1 (2013): 273-286.

[12] Hamdaqa, Mohammad, and Ladan Tahvildari. "Cloud computing uncovered: a research landscape." Advances in Computers 86 (2012): 41-85.

[13] Turskis, Zenonas, Edmundas Kazimieras Zavadskas, and Friedel Peldschus. "Multi- 
criteria optimization system for decision making in construction design and management." Engineering economics 61.1 (2015).

[14] Godse, Manish, and Shrikant Mulik. "An approach for selecting software-as-a-service (SaaS) product." Cloud Computing, 2009. CLOUD'09. IEEE International Conference on. IEEE, 2009.

[15] He, Qiang, et al. "QoS-driven service selection for multi-tenant SaaS." Cloud computing (cloud), 2012 ieee 5th international conference on. IEEE, 2012.

[16] Lo, Chi-Chun, et al. "Service selection based on fuzzy TOPSIS method." Advanced Information Networking and Applications Workshops (WAINA), 2010 IEEE 24th International Conference on. IEEE, 2010.

[17] Yazdani-Chamzini, Abdolreza, Siamak Haji Yakhchali, and Mahmood Mahmoodian. "Risk ranking of tunnel construction projects by using the ELECTRE technique under a fuzzy environment." International Journal of Management Science and Engineering Management 8.1 (2013): 1-14.

[18] Yu, Tao, and Kwei-Jay Lin. "Service selection algorithms for Web services with end-to-end QoS constraints." e-Commerce Technology, 2004. CEC 2004. Proceedings. IEEE International Conference on. IEEE, 2004.

[19]Zheng, Zibin, et al. "QoS ranking prediction for cloud services." IEEE transactions on parallel and distributed systems 24.6 (2013): 1213-1222.

[20] Ye, Zhen, Xiaofang Zhou, and Athman Bouguettaya. "Genetic algorithm based QoS-aware service compositions in cloud computing." International Conference on Database Systems for Advanced Applications. Springer Berlin Heidelberg, 2011.

[21] Wang, Shangguang, et al. "Particle swarm optimization with skyline operator for fast cloud-based web service composition." Mobile Networks and Applications 18.1 (2013): 116-121.

[22] Pan, Yuchen, et al. "Trust-enhanced cloud service selection model based on QoS analysis." PloS one 10.11 (2015): e0143448.

[23] Jøsang, Audun, Roslan Ismail, and Colin Boyd. "A survey of trust and reputation systems for online service provision." Decision support systems 43.2 (2007): 618-644.

[24] Jurca, Radu, Boi Faltings, and Walter
Binder. "Reliable QoS monitoring based on client feedback." Proceedings of the 16th international conference on World Wide Web. ACM, 2007.

[25]Chen, Cheng-Wu, et al. "Conceptual framework and research method for personality traits and sales force automation usage." Scientific Research and Essays 6.17 (2011): 3784-3793.

[26] Büyükyazıc1, Murat, and Meral Sucu. "The analytic hierarchy and analytic network processes." CRITERION 1 (2003): c1.

[27] Saaty, Thomas L. "The analytic network process." Decision making with the analytic network process. Springer, Boston, MA, 2006. 1-26

[28] Oran, Fatih Emre, et al. "A multi-criteria intuitionistic fuzzy group decision making for supplier selection with TOPSIS method." Expert Systems with Applications 36.8 (2009): 11363-11368.

[29] Figueira, José Rui, Vincent Mousseau, and Bernard Roy. "ELECTRE methods." Multiple Criteria Decision Analysis. Springer, New York, NY, 2016. 155-185.

[30] Afshari, Alireza, Majid Mojahed, and Rosnah Mohd Yusuff. "Simple additive weighting approach to personnel selection problem." International Journal of Innovation, Management and Technology 1.5 (2010): 511.

[31] DeVore, Ronald A., and Vladimir N. Temlyakov. "Some remarks on greedy algorithms." Advances in computational Mathematics 5.1 (1996): 173-187.

[32] Chen, Guo-chu, and Jin-shou Yu. "Particle swarm optimization algorithm." INFORMATION AND CONTROL-SHENYANG-34.3 (2005): 318.

[33]Zavadskas, Edmundas Kazimieras, Zenonas Turskis, and Simona Kildienè. "State of art surveys of overviews on MCDM/MADM methods." Technological and economic development of economy 20.1 (2014): 165179.

[34]Chen, Cheng-Wu, et al. "Conceptual framework and research method for personality traits and sales force automation usage." Scientific Research and Essays 6.17 (2011): 3784-3793.

[35] Büyükyazıc1, Murat, and Meral Sucu. "The analytic hierarchy and analytic network processes." CRITERION 1 (2003): c1.

[36] Godse, Manish, and Shrikant Mulik. "An approach for selecting software-as-a-service 
(SaaS) product." Cloud Computing, 2009. CLOUD'09. IEEE International Conference on. IEEE, 2009.

[37] Karim, Raed, Chen Ding, and Ali Miri. "An end-to-end QoS mapping approach for cloud service selection." Services (SERVICES), 203 IEEE Ninth World Congress on. IEEE, 2013.

[38] Gonçalves Junior, Ronaldo, et al. "A multicriteria approach for assessing cloud deployment options based on non-functional requirements." Proceedings of the 30th Annual ACM Symposium on Applied Computing. ACM, 2015.

[39] Menzel, Michael, Marten Schönherr, and Stefan Tai. "(MC2) 2: criteria, requirements and a software prototype for Cloud infrastructure decisions." Software: Practice and experience 43.11 (2013): 1283-1297.

[40] Sun, Le, et al. "Cloud-FuSeR: Fuzzy ontology and MCDM based cloud service selection." Future Generation Computer Systems 57 (2016): 42-55.

[41] Boutkhoum, Omar, et al. "Selection problem of cloud solution for big data accessing: fuzzy AHP-PROMETHEE as a proposed methodology." Journal of Digital Information Management 14.6 (2016).

[42] Afshari, Alireza, Majid Mojahed, and Rosnah Mohd Yusuff. "Simple additive weighting approach to personnel selection problem." International Journal of Innovation, Management and Technology 1.5 (2010): 511.

[43] Wachana Khowfa and Onsiri Silasai "The Integration of Association Rules and AHP in Cloud Service Selection". International Journal of Applied Engineering Research ISSN 0973-4562 Volume 12, Number 24 (2017) pp. 15814-15820.

[44] Y. Mohammadshahi, "A state -of-art survey on TQM applications using MCDM techniques, "Decision Science Letters, Vol2 no 3, pp. 125-134,2013 .

[45] Zavadskas, Edmundas Kazimieras, Algimantas Zakarevicius, and Jurgita Antucheviciene. "Evaluation of ranking accuracy in multi-criteria decisions." Informatica 17.4 (2006): 601618.

[46] Rai, Deepti, and P. Kumar. "Instance based multi criteria decision model for cloud service selection using TOPSIS and VIKOR." Int. J. Comput Eng. Technol 7 (2016): 78-87.

[47] Mohammadshahi, Yasaman. "A state-of-art survey on TQM applications using MCDM techniques." Decision Science Letters 2.3 (2013): 125-134.

[48]Fülöp, János. "Introduction to decision making methods." BDEI-3 workshop, Washington. 2005.

[49] Yazdani-Chamzini, Abdolreza, Siamak Haji Yakhchali, and Mahmood Mahmoodian. "Risk ranking of tunnel construction projects by using the ELECTRE technique under a fuzzy environment." International Journal of Management Science and Engineering Management 8.1 (2013): 1-14.

[50]Zheng, Zibin, et al. "QoS ranking prediction for cloud services." IEEE transactions on parallel and distributed systems 24.6 (2013): 1213-1222.

[51]He, Qiang, et al. "QoS-driven service selection for multi-tenant SaaS." Cloud computing (cloud), 2012 ieee 5th international conference on. IEEE, 2012

[52] Wang, Shangguang, et al. "Particle swarm optimization with skyline operator for fast cloud-based web service composition." Mobile Networks and Applications 18.1 (2013): 116-121.

[53] Kang, Guosheng, et al. "An effective dynamic web service selection strategy with global optimal QoS based on particle swarm optimization algorithm." Parallel and Distributed Processing Symposium Workshops \& PhD Forum (IPDPSW), 2012 IEEE 26th International. IEEE, 2012.

[54] Yang, Wanchun, et al. "A hybrid particle swarm optimization algorithm for service selection problem in the cloud." International Journal of Grid Distribution Computing 7.4 (2014): 1-10.

[55] Ye, Zhen, Xiaofang Zhou, and Athman Bouguettaya. "Genetic algorithm based QoS-aware service compositions in cloud computing." International Conference on Database Systems for Advanced Applications. Springer Berlin Heidelberg, 2011.

[56] Klein, Adrian, Fuyuki Ishikawa, and Shinichi Honiden. "Towards network-aware service composition in the cloud." Proceedings of the 21st international conference on World Wide Web. ACM, 2012.

[57]Tang, Maolin, and Lifeng Ai. "A hybrid genetic algorithm for the optimal constrained web service selection problem in web service composition." Evolutionary Computation (CEC), 2010 IEEE Congress 
on. IEEE, 2010.

[58] Pan, Yuchen, et al. "Trust-enhanced cloud service selection model based on QoS analysis." PloS one 10.11 (2015): e0143448.

[59] Jøsang, Audun, Roslan Ismail, and Colin Boyd. "A survey of trust and reputation systems for online service provision." Decision support systems 43.2 (2007): 618-644.

[60] Wu, Qingtao, et al. "Reputation revision method for selecting cloud services based on prior knowledge and a market mechanism." The Scientific World Journal 2014 (2014).

[61] Ghosh, Nirnay, Soumya K. Ghosh, and Sajal K. Das. "SelCSP: A framework to facilitate selection of cloud service providers." IEEE transactions on cloud computing 3.1 (2015): 66-79.

[62] Ma, Hua, et al. "Toward trustworthy cloud service selection: A time-aware approach using interval neutrosophic set." Journal of Parallel and Distributed Computing 96 (2016): 75-94.

[63] Supriya, M., K. Sangeeta, and G. K. Patra. "Trustworthy Cloud Service Provider Selection using Multi Criteria Decision Making Methods." Engineering Letters 24.1 (2016).

[64] Jurca, Radu, Boi Faltings, and Walter Binder. "Reliable QoS monitoring based on client feedback." Proceedings of the 16th international conference on World Wide Web. ACM, 2007.

[65] Jurca, Radu, and Boi Faltings. "An incentive compatible reputation mechanism." ECommerce, 2003. CEC 2003. IEEE International Conference on. IEEE, 2003.

[66] Papaioannou, Thanasis G., and George D. Stamoulis. "A mechanism that provides incentives for truthful feedback in peer-topeer systems." Electronic Commerce Research 10.3-4 (2010): 331-362.

[67] Achar, Raghavendra, and P. Santhi Thilagam. "A broker based approach for cloud provider selection." Advances in Computing, Communications and Informatics (ICACCI, 2014 International Conference on. IEEE, 2014.

[68] Siegel, Jane, and Jeff Perdue. "Cloud services measures for global use: the Service Measurement Index (SMI)." SRII Global Conference (SRII), 2012 Annual. IEEE, 2012.

[69] Srivastava, Abhishek, and Paul G. Sorenson. "Service selection based on customer rating of quality of service attributes." Web Services (ICWS), 2010 IEEE International Conference on. IEEE, 2010.

[70] Callaghan, Alison, and Kemper Lewis. "A 2-phase aspiration-level and utility theory approach to large scale design." Proceedings of the ASME DETC 2000 (2000).

[71]Michael Kulok and Kemper Lewis, Preference consistency in multiattribute decision making, Proceedings of Design Engineering Technical Conferences And Computers and Information in Engineering Conference, 2005.

[72] Sundareswaran, Smitha, Anna Squicciarini, and Dan Lin. "A brokerage-based approach for cloud service selection." Cloud Computing (CLOUD), 2012 IEEE 5th International Conference on. IEEE, 2012.

[73] Pandey, Sarvesh, and A. K. Daniel. "QoCS and Cost Based Cloud Service Selection Framework." International Journal of Engineering Trends and Technology (IJETT) - Volume 48 Number 3 June 2017 